\title{
Evaluation of clinical and laboratory causes of burns in pre- school children
}

\author{
Mohammadreza Mobayen¹, Siamak Rimaz ${ }^{1}$, Arghavan Malekshahi1, ${ }^{*}$ \\ ${ }^{1}$ Burn and Regenerative Medicine Research Center, Guilan University of Medical Sciences, Rasht, Iran
}

\begin{abstract}
We investigate characteristics of burns in children aged up to seven years and hospitalized at Velayat burn center unit for two years to evaluate clinical and laboratory causes of burns in pre-school children. In a retrospective study, all patients under 6 years who were admitted to Velayat hospital due to burn injuries between 20 march 2016 and 20 march 2017 participated in this study. Baseline data and information collected from hospital HIS system and patient records. The burn-injured children were 283 patients, of which $54.1 \%$ were male and $45.9 \%$ were female. These injuries mostly were caused by hot liquid $(78.7 \%)$. The greatest number of burn victims were boys between one and two years old. Almost all the accidents occurred in the home environment (90.1\%). A significant correlation was observed between contact burns, serum albumin level, and serum C-reactive protein (CRP) levels with burn severity. Our data demonstrate the importance of developing a program for the prevention of pediatric scalds with the education of family members to be aware of the danger. The most important finding in our study was the hypothesis of estimating Albumin 1b peptides (A1b) and CRP laboratory factors during the initial week of hospitalization in the prognosis of children and their constant relation with the severity level of the burns.
\end{abstract}

Keywords: Burn injuries, Pediatric, Epidemiologic, Laboratory

\section{Introduction}

Burn injuries continue to be one of the leading causes of injuries worldwide [1]. In addition, a considerable level of hospital care is attributed to burns annually [2]. According to reports, 95\% of burnrelated deaths occur in low- and middle-income countries. In fact, half of the death cases are observed in South and Southeast Asia [2, 3]. Therefore, burns are predictable injuries in children [4], which, at the same time, could lead to numerous destructive physical, mental and social issues [5].

In addition to their acute impacts, burns can cause chronic disorders in the lives of children [6]. Children are highly vulnerable to burn-related injuries. While

\footnotetext{
* Corresponding author:

Arghavan Malekshahi, MD

Burn and Regenerative Medicine Research Center,

Poursina Hospital, Namjoo Street, Rasht, Iran

Tel/Fax: +9813 333311473

Email: arghavan.malekshahi.am@gmail.com

https://orcid.org/o0oo-0002-7102-0653
}

Received: January, 09, 2020

Accepted: February, 16, 2020 infants and pre-school children are more at risk of burn injuries, they are more neglected, compared to other age groups [7, 8]. Moreover, studies have shown that more than $50 \%$ of burns occur in children aged three-six years $[9,10]$. Given the high incidence rate of burns in children aged one to six years and the high rate of associated complications and mortality rate in children. We aimed to evaluate clinical and laboratory causes of burns in pre-school children.

\section{Materials and Methods}

The research was performed on patients hospitalized from March 2016 to March 2017 due to different burn-related injuries with various 
mechanisms. Burn mechanisms are divided into three thermal (heat-related), chemical, electrical categories. Thermal burns include flame, hot liquids, and contact with hot surfaces. In this regard, not only flame burns are the most common causes of burn-related hospitalizations, but also, they have the highest mortality rate, which is primarily due to their association with building fires and respiratory impairments or carbon monoxide (CO) poisoning. The burned area is estimated using the rule of nines; in this respect, $18 \%$ for each anterior and posterior trunk, $9 \%$ for each upper limb, $18 \%$ for each lower limb, $9 \%$ for head and neck, and $1 \%$ for perineum are estimated. Based on depth, burns are classified into first-degree (superficial), second-degree (partial thickness), thirddegree (full thickness), and fourth-degree burns, which go through both layers of the skin and underlying tissue as well as deeper tissue.

The inclusion criteria were patients experiencing burns with different mechanisms and the age range of zero to six years. On the other hand, the exclusion criterion was incompletemedical records. Using a predesigned questionnaire, we collected the data related to demographic characteristics of the subjects, including age, gender, cause of the burn, the season of burn, the anatomical site of the burn, burn site, mortality, duration of hospitalization, burn severity (percentage), burn degree, CRP serum level, and albumin (in the first seven days of hospitalization). The required information was extracted from patients' records and their history and the data were entered into SPSS software ver. 22. It should be noted that patients who have been hospitalized more than once were considered as one patient and the information about several hospitalizations was combined and analyzed as one patient. Ordered logistic regression was used to investigate the relationship between different variables and burn severity. The poisson regression model was also used to investigate the relationship between burn percentage and different variables.

\section{Results}

A total of 283 patients entered the study aiming to examine the epidemiology of annual cases of burns in preschool children in Velayat hospital. Out of the 283 studied patients, 153 (54.1\%) patients were male and 129 (45.9\%) were female. The average age of the patients was $2.1 \pm 1.6$ years (the youngest patient being an infant and the oldest being 6 years old). The study of the time of incident indicated that most of the cases of burns had occurred in July with 32 cases (11.3\%) and the lowest number of cases was observed in September with 14 cases (4.9\%); however, the largest number of cases had occurred during winter (35.4\%). The study of patients' place of residence divided by urban and rural areas indicated that out of the studied population, 219 people $(77.4 \%)$ lived in the city while 64 patients (22.6\%) lived in rural areas. Evaluation of the reasons for burning indicated that over half of the patients $(78.7 \%)$ had burned themselves with hot liquids including hot tea and water, stew, or soup. Also, one patient had burned due to a gas explosion, six patients had burned due to contact with fire and alcohol, and 87 patients (30.7\%) had suffered from other sources of burning such as contact with heaters, and hot objects. Investigation of the burn site revealed that 99 patients (35\%) had burns on several areas including the upper body and limbs. Also, 82 patients had burns on their lower body, and 65 patients had burns on their upper body. Besides, 23 patients suffered from burns on their torso, and 13 patients had burns on their face and head. Investigation of the degree of burns indicated that 58 patients had suffered from first-degree burns, 173 patients suffered from second-degree burns, and four patients suffered from third-degree burns. Besides, 48 patients suffered from moderate burns ranking between second-degree and third-degree burns. Examination of patients' burn percentage revealed that 32 patients with $5 \%$ burns, 27 patients with $6 \%$ burns, and 19 patients with $20 \%$ burns had the most frequent burn percentage among the study population. Examination of the hospitalization period indicated that over half (53\%) of patients were treated on an outpatient basis and discharged. Besides, 54 patients were hospitalized for one day and 22 patients were hospitalized for two days. The study of burning consequences revealed that only one patient had died due to burns and other patients had been discharged. The study of wound culture in patients indicated that eight cultures (2.8\%) tested positive and the rest tested negative.

Table 1 demonstrates the studied patient's serum albumin levels on days zero, three, and seven, and serum CRP levels on days zero and seven.

Table 2 demonstrates study results based the relationship between burn intensity and various order logistic variables used in the study. Given that burn 
Table1. Serum albumin levels on days 0,3 and 7 and serum CRP levels on days 0 and 7

\begin{tabular}{lcccc}
\hline Variable & Minimum & Maximum & Mean & Std. Deviation \\
\hline Albumin baseline (gr/dl) & 1.50 & 4.50 & 2.64 & 0.80 \\
\hline Albumin day 3 (gr/dl) & 1.80 & 4.00 & 2.73 & 0.54 \\
\hline Albumin day 7 (gr/dl) & 2.00 & 4.50 & 3.01 & 0.68 \\
\hline CRP baseline (mgr/dl) & 0 & 125.00 & 13.50 & 24.21 \\
\hline CRP day 7 (mgr/dl) & 0 & 150.00 & 10.03 & 19.21 \\
\hline
\end{tabular}

Table 2. Examines the relationship between burn severity and other variables

\begin{tabular}{|c|c|c|c|c|c|}
\hline Burn variable & Odds Ratio & Standard error & P-value & Low limit & High limit \\
\hline Age & 1.061 & 0.087 & 0.473 & 0.903 & 1.246 \\
\hline (Girl to boy) sex & 0.579 & 0.162 & 0.51 & 0.335 & 1.003 \\
\hline \multicolumn{6}{|l|}{ Season } \\
\hline Summer & 2.028 & 1.295 & 0.268 & 0.580 & 7.087 \\
\hline Fall & 1.240 & 0.785 & 0.734 & 0.358 & 4.290 \\
\hline Winter & 1.137 & 0.722 & 0.840 & 0.328 & 3.947 \\
\hline \multicolumn{6}{|l|}{ Reference: Spring } \\
\hline \multicolumn{6}{|l|}{ Cause of burns } \\
\hline Explosion & 0.855 & 0.918 & 0.990 & $<0.001$ & 2.125 \\
\hline Fire & 0.855 & 0.785 & 0.865 & 0.142 & 5.168 \\
\hline Contact burns & 0.025 & 0.011 & $<0.001$ & 0.011 & 0.058 \\
\hline \multicolumn{6}{|l|}{ Reference: Electric burn } \\
\hline \multicolumn{6}{|c|}{ Anatomical location of the burn } \\
\hline Body & 1.441 & 1.154 & 0.648 & 0.300 & 6.920 \\
\hline Upper limb & 2.419 & 1.683 & 0.204 & 0.619 & 9.458 \\
\hline Lower limbs & 1.585 & 1.090 & 0.503 & 0.412 & 6.099 \\
\hline Several limbs together & 2.316 & 1.596 & 0.223 & 0.600 & 9.941 \\
\hline Other anatomical places & 0.352 & 0.863 & 0.670 & 0.003 & 42.932 \\
\hline \multicolumn{6}{|l|}{ Reference: face } \\
\hline \multicolumn{6}{|l|}{ Burn site } \\
\hline Bathroom & 0.373 & 0.653 & 0.573 & 0.012 & 11.537 \\
\hline School & $<0.001$ & $<0.001$ & 0.983 & $<0.001$ & 1.203 \\
\hline Nature & 2.279 & 1.322 & 0.155 & 0.731 & 7.104 \\
\hline Bedroom & 1.824 & 1.310 & 0.403 & 0.446 & 7.455 \\
\hline \multicolumn{6}{|l|}{ Reference: Kitchen } \\
\hline \multicolumn{6}{|l|}{ Laboratory parameters } \\
\hline Serum albumin baseline & 0.014 & 0.01 & $<0.001$ & 0.01 & 0.048 \\
\hline Serum albumin day 3 & 0.01 & 0.012 & $<0.001$ & 0.02 & 0.045 \\
\hline Serum albumin level day 7 & 0.012 & 0.08 & $<0.001$ & 0.01 & 0.041 \\
\hline Serum CRP level baseline & 0.01 & 0.01 & $<0.001$ & 0.011 & 0.035 \\
\hline Serum CRP level 7 & 0.013 & 0.01 & $<0.001$ & 0.012 & 0.033 \\
\hline
\end{tabular}

severity indicated in this table is an interval variable, sequential logistic regression model has been used. Therefore, the coefficients in this table are odds ratio. According to this table, a significant correlation was observed between contact burns, serum albumin level (in all three days), and serum CRP levels (in both examinations) with burn severity. 


\section{Discussion}

Burning injuries are considered to be one of the most common types of injuries in the entire world [1113], besides, injuries in children being a significant percentage and a major part of hospital care annually $[14,15]$. During the studies of the precise burn percentage of burnt patients which was conducted on the population who were referring to our hospital, it was discovered that the majority of these patients had approximately 5\% burning injury overall, which is significantly lower than the burn percentage of similar studies conducted previously in Turkey and India [6, 16].

In the study of the outcome of burn patients who referred to our hospital, it was discovered that only one patient's burning injuries were fatal and the rest were all discharged from the hospital, which was considerably less than the number of fatally injured and burnt patients found in other parts of Iran such as, Isfahan and Mashhad. In Isfahan, it was discovered that $5.6 \%$ of burns resulted in death, and in Mashhad, the mortality rate was about $3.85 \%[1,17]$.

Evaluation on the hospitalization period shows that the duration of hospitalization of burn patients who were referring to our hospital is approximately one or two days on average, which is significantly less than the results obtained from a study conducted in Turkey [6]. In another study conducted under quite similar circumstances in Turkey, the average number of days burnt patients were hospitalized was approximately 16 days [4]. In another study conducted in Iran (Isfahan), patients averaged about less than three days in hospital until released [1].

In the process of studying the wound cultures of our hospital burn patients, it was discovered that the wound culture samples of eight of these patients were positive and the rest all turned out negative, which was lower than other studies $[6,17]$.

Based on the studies done so far, it can be concluded that most of these burn injuries occur in the home, especially in the kitchen, also, most of these fires occur due to the absence of parents and children being left alone [18], which can lead to many possible physical and psychological consequences, including dissatisfaction with body shape, post-accident trauma, and stress disorder, as well as a sleep disorder, wound and scar issues and on the other hand. It can also impose great costs both financially as well as mentally for the surrounding community and relatives of the individual in terms of fixing the deformities created, in addition to treating and compensating all the psychological issues in the aftermath of the burns. Therefore, the prevention of burns is always preferred and much better than being cured, fortunately, there have been many positive reports recently about the reduction of burns and mortality rate due to the population following the fire prevention programs [19]. As a consequence, having either a proper parental awareness program through the mass media and TV or properly educating parents about the level of care and cautiousness the children under the age of six need can lead to a reduction in fire occurrences and all the unpleasant consequences that may follow afterward.

In our study, we discovered that the precise serum albumin levels during the first week as well as CRP in this group of children can be a factor accompanying the severity level of burns and it is for this purpose that these two criteria can be used as a useful predictor for the prognosis of burns. Although we were not able to investigate any further due to the existence of only one case of mortality in our study, therefore, it is recommended to examine this particular hypothesis in upcoming future studies.

The main limitations of the present study were due to incomplete files and incomplete entry of findings, some samples were excluded from the study. Also, our results are limited only to two years which can limit the strength of our conclusion.

According to the various study results obtained based on the high frequency of burn injuries in children under the age of six which usually seems to take place in their homes and kitchens and can potentially lead to death, scar issues and depression, etc. This can only indicate the extreme level of importance taking proper care of children can have, as well as the constant presence of the parents required who are prepared and aware of the potential dangers to prevent such disastrous incidents. However, the most important and major finding in our study so far was the hypothesis of estimating A1b and CRP laboratory factors during the initial week of hospitalization in the prognosis of children and their constant relation with the severity level of the burns. 


\section{Authors Contribution}

All authors contributed equally in data collection and drafting of the manuscript. Also, all authors approved the final version of the manuscript.

\section{Conflict of interests}

The authors do not have any conflict of interest to disclose.

\section{Ethical declarations}

The study deign was approved by the ethics committee of Guilan University of Medical Sciences (IR.GUMS.REC.1397.165).

\section{Financial Support}

None.

\section{References}

1. Ahmadabadi A, Tavousi SH, Sedaghat A, Khadem Rezaeyan M, Yaghoubi Moghaddam Z, Lalavi Z. Pattern of burn injuries in preschool children. Saf Promot Inj Prev. 2016; 4(4):225-30.

2. Seifert J. Incidence and economic burden of injuries in the United States. BMJ Publishing Group Ltd; 2007.

3. Khadem-Rezaiyan M, Aghajani H, Ahmadabadi A, Zanganeh M, Tavousi SH, Sedaghat A, et al. Epidemiology of severe burns in North-East of Iran: How is the burn size different in a developing country from developed ones? Burns Open. 2020; 4(1):4-9.

4. Anlatici R, Ozerdem OR, Dalay C, Kesiktaş E, Acartürk S, Seydaoğlu G. A retrospective analysis of 1083 Turkish patients with serious burns. Part 2: burn care, survival and mortality. Burns. 2002; 28(3):239-43.

5. De Young AC, Kenardy JA, Cobham VE, Kimble R. Prevalence, comorbidity and course of trauma reactions in young burn-injured children. J Child Psychol Psychiatry. 2012; 53(1):56-63.

6. Balseven-Odabaşi A, Tümer AR, Keten A, Yorganci K. Burn injuries among children aged up to seven years. Turk $\mathrm{J}$ Pediatr. 2009; 51(4):328-35.

7. Shields BJ, Comstock RD, Fernandez SA, Xiang H, Smith GA. Healthcare resource utilization and epidemiology of pediatric burn-associated hospitalizations, United States, 2000. J Burn Care Res. 2007; 28(6):811-26.

8. Carlsson A, Udén G, Håkansson A, Karlsson ED. Burn injuries in small children, a population-based study in Sweden. J Clin Nurs. 2006; 15(2):129-34.

9. Libber SM, Stayton DJ. Childhood burns reconsidered: the child, the family, and the burn injury. J Trauma. 1984; 24(3):24552 .

10. Kent L, King H, Cochrane R. Maternal and child psychological sequelae in paediatric burn injuries. Burns. 2000; 26(4):317-22.

11. Ghosh A, Bharat R. Domestic burns prevention and first aid awareness in and around Jamshedpur, India: strategies and impact. Burns. 2000; 26(7):605-8.

12. Wiechman SA, Ptacek JT, Patterson DR, Gibran NS, Engrav LE, Heimbach DM. Rates, trends, and severity of depression after burn injuries. J Burn Care Rehabil. 2001; 22(6):417-24.
13. Forjuoh SN. Burns in low- and middle-income countries: a review of available literature on descriptive epidemiology, risk factors, treatment, and prevention. Burns. 2006; 32(5):529-37.

14. Kazemzadeh J, Vaghardoost R, Dahmardehei M, Rabiepoor S, Farzan R, Asghar Kheiri A, et al. Retrospective Epidemiological Study of Burn Injuries in 1717 Pediatric Patients: 10 Years Analysis of Hospital Data in Iran. Iran J Public Health. 2018; 47(4):584-90. 15. Kao CC, Garner WL. Acute Burns. Plast Reconstr Surg. 2000; 101(7):2482-93.

16. Verma S, Srinivasan S, Vartak A. An epidemiological study of 500 paediatric burn patients in Mumbai, India. Indian J Plast Surg. 2007; 4O(2):153-7.

17. Rafii MH, Saberi HR, Hosseinpour M, Fakharian E, Mohammadzadeh M. Epidemiology of pediatric burn injuries in isfahan, iran. Arch Trauma Res. 2012; 1(1):27-30.

18. Joseph KE, Adams CD, Goldfarb IW, Slater H. Parental correlates of unintentional burn injuries in infancy and early childhood. Burns. 2002; 28(5):455-63.

19. Van der Merwe AE, Steenkamp WC. Prevention of burns in developing countries. Ann Burns Fire Disasters. 2012; 25(4):18891. 